\title{
Magnetorheological Properties of Ferrofluids Containing Clustered Particles
}

\author{
A. Yu. Zubarev and D. N. Chirikov \\ Ural Federal University, ul. Lenina 51, Yekaterinburg, 620000 Russia
}

Received December 11, 2012

\begin{abstract}
A theoretical model is proposed to describe experimental data on the magnetorheological properties of magnetic fluids containing clustered particles consisting of single-domain ferromagnetic nanoparticles distributed in a polymeric shell $80-100 \mathrm{~nm}$ in diameter. These fluids combine the sedimentation stability typical of nanodisperse ferrofluids with the high sensitivity of rheological parameters to magnetic fields. The developed model explains the experimentally found long-term rheological relaxation and residual stress that is retained after the medium ceases to flow.
\end{abstract}

DOI: $10.1134 / \mathrm{S} 1061933 \mathrm{X} 13050190$

\section{INTRODUCTION}

The possibility of controlling the properties and behavior of fluids and polymers by means of magnetic fields allows one to obtain new solutions for a number of applied and engineering problems. All natural liquid media are diamagnetic; hence, they interact very poorly with magnetic fields. Magnetic control over their behavior requires the generation of strong fields, which is associated with significant technological difficulties. The possibility of using fairly weak and moderate fields, the generation of which requires no bulky and expensive setup, is of greatest interest for practically important applications. Artificially synthesized suspensions of magnetic particles, which are usually referred to as magnetic fluids (ferrofluids), are materials that can be easily controlled in weak fields.

The particle diameter in typical ferrofluids varies within 7-20 $\mathrm{nm}$. Strong magnetorheological effects are only observed in these systems at low shear rates [1]. In suspensions of microsized magnetic particles, strong rheological effects are observed within a broad range of shear rates; however, these suspensions are unstable with respect to sedimentation. Rather rapid sedimentation of massive non-Brownian particles onto the bottom of a container degrades the technological properties of these suspensions. The problem of synthesizing magnetic fluids that would combine the sedimentation stability of typical ferrofluids with pronounced magnetorheological properties has existed for rather a long time. Magnetic fluids consisting of iron-oxide nanoparticles assembled into clusters $80-100 \mathrm{~nm}$ in diameter by means of polymer shells have turned out to comply with these requirements [2, 3 ]. Clustered magnetic fluids are a promising material for a number of modern technologies.

Figure 1 shows a schematic representation of magnetic clusters. Each cluster consists of single-domain nanoparticles distributed in a polymer shell. Micrographs of these clusters have been given in [2]. Ferromagnetic nanoparticles form the magnetic core of a cluster, which is surrounded with a pure polymer layer. It should be mentioned that the scheme shown in Fig. 1 is a strongly idealized one. Real clusters have irregular shapes and broad size distributions. The average diameter of a cluster with a polymer shell is $\sim 80$ $100 \mathrm{~nm}$, while the size of the magnetic core is $\sim 50$ $70 \mathrm{~nm}$. Volume fraction of a magnetic material $\varphi$ in clustered magnetic fluids that have been synthesized at present is approximately $0.1 \%[2,3]$.

Experiments conducted with stationary flows of clustered magnetic fluids [3] have showed that the viscosity of these systems can increase by approximately

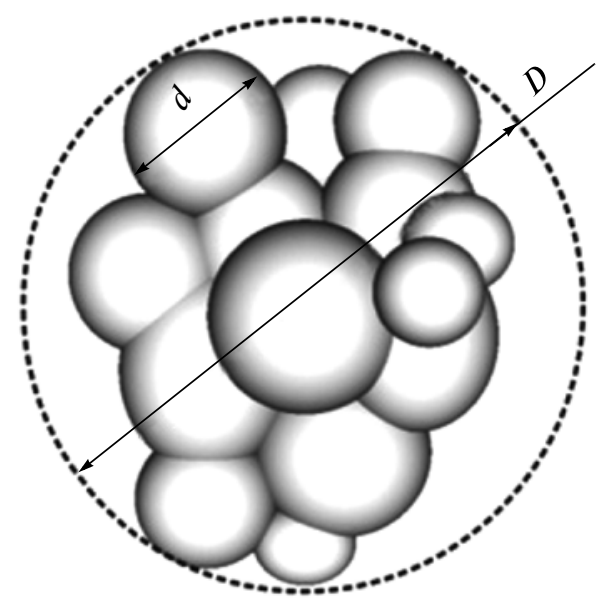

Fig. 1. Schematic representation of a magnetic cluster: grain diameter, $d=10-30 \mathrm{~nm}$; cluster diameter, $D=50$ $70 \mathrm{~nm}$. 
two orders of magnitude in magnetic fields. Data on the shear stress relaxation in clustered fluids after a sudden change in the shear flow rate have been reported in [4]. The experiments were carried out in a rotational rheometer equipped with a cone-plane measuring unit, into which a magnetic fluid was placed. The design and principle of operation of such rheometers can be found in [1].

In the experiments, magnetic field $\mathbf{H}$ was imposed in parallel to the rotation axis of the rheometer, i.e., in the direction of the flow-velocity gradient of the magnetic fluid. The design of the rheometer made it possible to promptly start and stop the cell cone, i.e., to force the fluid to either begin or stop flowing almost instantaneously.

Figure 2a illustrates the experimental time dependences of shear stress $\sigma$ measured after a clustered magnetic fluid is stopped at different magnetic field strengths. The experimental data show a long-term stress relaxation with a characteristic time of several dozens and even hundreds of seconds. Note that the relaxation times of ordinary ferrofluids with the same low concentrations of the magnetic phase are approximately $10^{-5}-10^{-4} \mathrm{~s}[5,6]$. The existence of a residual stress is an unexpected effect that has been revealed in these experiments and was not observed for the other types of magnetic fluids. As can be seen from the time dependences of the stress (Fig. 2a), after the magnetic fluid ceases to move, stress $\sigma$ tends to a nonzero final value. Residual stress $\sigma_{\mathrm{r}}$ remained preserved in the course of the experiments, which lasted several hours.

The goals of this work were to theoretically explain the physical nature of the observed relaxation phenomena and develop a model for the rheological properties of the clustered ferrofluid.

\section{PHYSICAL MODEL}

Strong rheological effects in magnetic fluids usually arise due to the aggregation of particles into heterogeneous structures stretching along the applied magnetic field (e.g., see [1, 6-9]). These structures typically look like linear chains or large droplets. Unfortunately, there is no reliable theory that would allow one to answer the question of the conditions under which chains rather than large clusters arise in a magnetic fluid.

The problem becomes still more complex for the clustered fluids under consideration due to the irregular shape and polydispersity of the clusters, as well as a rather wide size distribution. In order to maximally simplify the analysis and obtain physically observable results, let us consider the model of identical spherical clusters shown in Fig. 1.

The stationary magnetoviscous properties of clustered magnetic fluids were quantitatively described in [3] based on a model that implied the aggregation of clustered particles into linear chains with their lengths
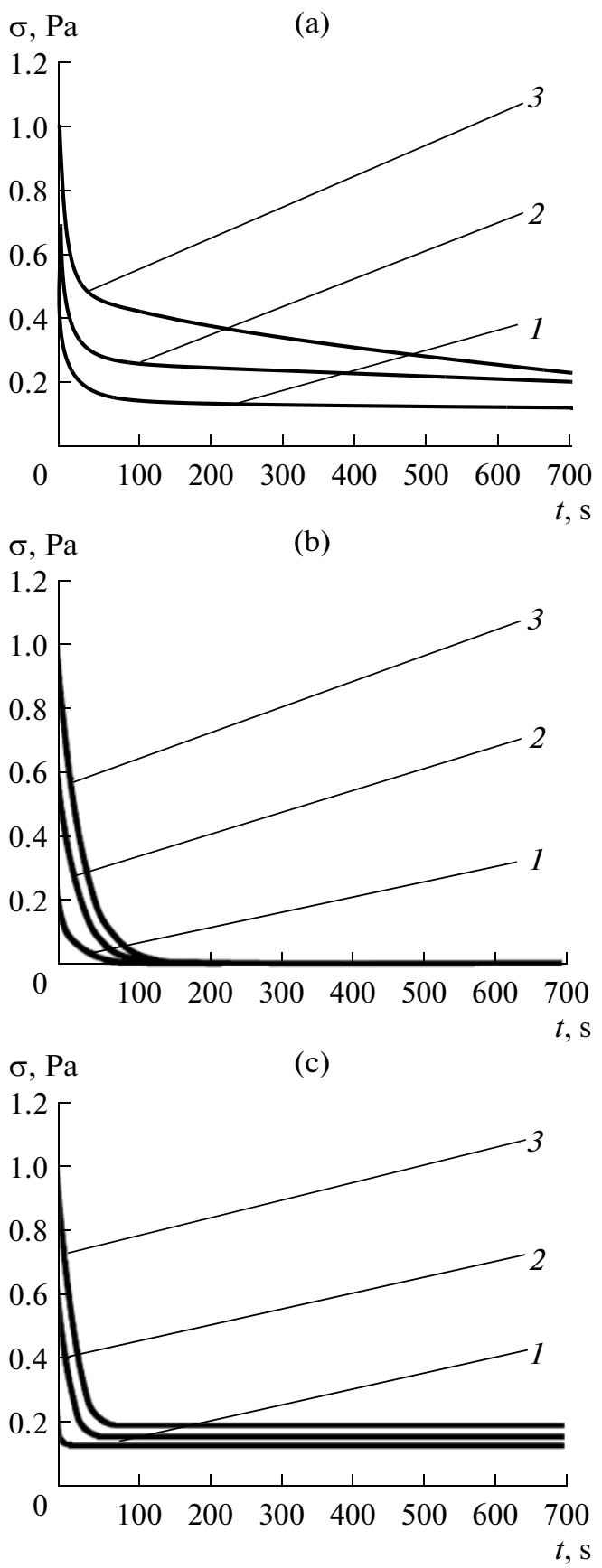

Fig. 2. (a) Experimental and (b, c) theoretical time dependences of stress at different magnetic field strengths $H=$ (1) 15 , (2) 20 , and (3) $30 \mathrm{kA} / \mathrm{m}$; (b, c) calculations by Eq. (8) (c) with allowance for the residual stress. Shear rate $\gamma$ varies in a range of $0.02-0 \mathrm{~s}^{-1}$, the viscosity of the carrier liquid is $\eta_{0}=0.1 \mathrm{~Pa} \mathrm{~s}$, the hydrodynamic cluster diameter is $D_{\mathrm{h}}=90 \mathrm{~nm}$, the hydrodynamic particle diameter is $d_{\mathrm{h}}=$ $21.5 \mathrm{~nm}$, and the magnetic diameter of a particle is $d_{\mathrm{m}}=$ $17.5 \mathrm{~nm}$.

being considerably shorter than the thickness of a measuring unit (i.e., the channel in which the magnetic fluid flows). Therefore, here we also assume that such chains predetermine macroscopic stress $\sigma$ at 
specified shear rate $\dot{\gamma}$, as well as the stress relaxation after the fluid flow is stopped.

Meanwhile, it is clear from physical considerations that a constant residual stress can emerge only under the condition that some structures block the flow in channel and interconnect the opposite walls of the channel after the movement of the channel wall is stopped. If the axes of these structures are tilted with respect to an external field, the arising moments of the magnetic forces tending to rotate these axes toward the orientation along the field will generate a mechanical force applied to the channel walls. The physical nature of the residual stress must be identical to the nature of the development of elastic shear forces in a magnetic suspension placed into an external field. The review of the studies devoted to describing the elasticity effects of magnetic suspensions and determining their static yield stresses can be found in [9].

The gaps in the measuring units of rotational rheometers are $1-2 \mathrm{~mm}$ wide. Since a clustered particle is $\sim 100 \mathrm{~nm}$ in diameter, a linear chain blocking the measuring unit is believed to contain more than 10 thousand particles. It is unrealistic that such long chains can be formed. Hence, static residual stresses can only result from the formation of dense large aggregates. These aggregates have been repeatedly observed in various magnetic fluids and suspensions $[9,10]$ placed into external magnetic fields.

Thus, experimental data $[3,4]$ indicate that clustered particles may aggregate into both linear chains and dense large "droplets." Aggregates of the first type are responsible for the magnetoviscous and relaxation effects under the conditions of a developed flow of the medium, while the aggregates of the second type give rise to the appearance of static residual stress. The formation of different types of aggregates can be related to the polydispersity of clustered particles. In an external magnetic field, the energy of magnetic interaction between the majority of clusters can be sufficient to cause the formation of chains but insufficient for the appearance of large aggregates. The interaction between relatively few large clusters can be sufficiently strong to provide formation of dense bulky structures. This point of view is supported by the known fact that, as the magnetic field strength is elevated, the initial formation of linear chains is followed by their condensation into dense phases (see $[9,11])$.

\section{CHAIN-SHAPED AGGREGATES}

In this section, we shall consider chain-shaped aggregates resulting from coalescence of magnetized clustered (composite) particles.

By neglecting the interaction between nanoparticles in a composite particle, its magnetic moment $m_{\mathrm{c}}$ can, as a first approximation, be estimated using the Langevin formula:

$$
m_{\mathrm{c}}=N_{\mathrm{p}} m_{\mathrm{p}} L\left(\frac{\mu_{0} m_{\mathrm{p}} H}{k T}\right), \quad L(x)=\operatorname{cth}(x)-\frac{1}{x} .
$$

where $N_{\mathrm{p}}$ is the number of nanoparticles in the magnetic core of a cluster, $m_{\mathrm{p}}$ is the magnetic moment of a particle, $L(x)$ is the Langevin function, $\mu_{0}$ is the magnetic permeability of vacuum, $H$ is the magnetic field strength, and $k T$ is the absolute temperature expressed in energy units.

Strictly speaking, this formula is believed to comprise the magnetic field inside a cluster. However, the internal field cannot be reliably calculated for irregularly shaped clusters. Calculation of this field for a model spherical cluster is cumbersome and yields uncontrollable divergence between the calculated field and the field inside a real irregularly shaped cluster. Therefore, we shall consider field $H$ as an averaged magnetic field in the ferrofluid in order to maximally simplify the calculation. Since, in the case under consideration, concentration $\varphi$ of ferromagnetic particles is low, the field inside the ferrofluid is approximately equal to the imposed external field.

Langevin formula (1) for the magnetization of an ensemble of ferromagnetic particles is exact when the interparticle magnetic interaction is negligible. It is well known that this formula underestimates the magnetization of concentrated systems of interacting ferroparticles. Nevertheless, in order to avoid cumbersome expressions when estimating the viscosity of a ferrofluid and obtain estimates of the magnetization of the cluster core with an accuracy on the order of magnitude, we use the simplest Langevin law (1).

Number $N_{\mathrm{p}}$ of nanoparticles in a cluster can be estimated as

$$
N_{\mathrm{p}}=\frac{\varphi_{\mathrm{p}} V_{\mathrm{m}}}{v_{\mathrm{h}}} .
$$

where $\varphi_{\mathrm{p}}$ is the particle volume concentration in the magnetic core of a cluster; $V_{\mathrm{m}}$ is the volume of a cluster magnetic core; and $v_{h}$ is the hydrodynamic volume of a nanoparticle as determined with allowance for the stabilizing surface layers, which prevent the particles from coalescence under the action of dispersion forces.

The electron micrographs [2] indicate a dense arrangement of nanoparticles in the core of a clustered particle. This fact allows us to use estimate $\varphi_{\mathrm{p}} \approx 0.5-$ 0.6 .

The estimates indicate that the interaction between the magnetized clusters and imposed field $H$ is considerably stronger than the interaction between the clusters in a chain. This is why, similarly to many other models of chains in magnetic suspensions (see, e.g., [8], and review [9]), it is assumed that magnetic 
moment $m_{\mathrm{c}}$ of a clustered particle in a chain is equal to the moment of an individual one. In other words, the effect of the magnetic interaction between clustered particles on their magnetic moments is neglected.

In compliance with approach [12], let us consider a chain consisting of magnetizable clusters, the magnetic moments of which are oriented along the imposed field (Fig. 3). Under the action of hydrodynamic shear forces, the axis of the chain deviated from the field direction by angle $\theta$.

After the flow is stooped, i.e., at $\gamma=0$, the dependence of total macroscopic shear stress $\sigma$ on time $t$ in the suspension of the chains can be represented as follows [13, 14]:

$$
\begin{gathered}
\sigma(t)=\sigma^{\mathrm{s}}(t)+\sigma^{\mathrm{a}}(t), \\
\sigma^{\mathrm{a}}(t)=\frac{1}{2} \sum_{n=2}^{n_{\mathrm{c}}} \Gamma_{n}^{\mathrm{m}}(t) g_{n}, \\
\sigma^{\mathrm{s}}(t)=-\eta_{0} V_{\mathrm{h}} \sum_{n=2}^{n_{\mathrm{c}}} \beta_{n} \frac{d}{d t}\left[\sin \theta_{n}(t) \cos \theta_{n}(t)\right] n g_{n},
\end{gathered}
$$

where

$$
V_{\mathrm{h}}=\frac{\pi D_{\mathrm{h}}^{3}}{6} .
$$

Here, $\sigma^{\mathrm{s}}$ and $\sigma^{\mathrm{a}}$ are the symmetric and antisymmetric components of the stress, respectively; $n_{\mathrm{c}}$ is the maximum number of clusters in an intact chain; $\Gamma_{n}^{\mathrm{m}}$ is the moment of magnetic force acting on the chain; $g_{n}$ is the number of $n$-particle chains per unit volume; $\eta_{0}$ is the viscosity of the carrier liquid; $V_{\mathrm{h}}$ is the hydrodynamic volume of a cluster; $D_{\mathrm{h}}$ is the hydrodynamic diameter of the cluster; $\beta_{n}$ is a coefficient determined in the Appendix; and $\theta_{n}$ is the angle between an $n$-particle chain and the direction of the external magnetic field axis.

Maximum number of clusters in an intact chain $n_{\mathrm{c}}$ is determined, first, by the balance between force $F_{\mathrm{r}}^{\mathrm{m}}$ of the magnetic attraction between the clusters in the chain and hydrodynamic force $F_{\mathrm{r}}^{\mathrm{h}}$, that disrupts this chain. Second, it is determined by the balance between hydrodynamic moment $\Gamma_{n}^{\mathrm{h}}$, that tends to cause deviation of the chain from the direction of the magnetic field toward the direction of the medium flow and magnetic moment of force $\Gamma_{n}^{\mathrm{m}}$ that tends to orient the chain along the field. Analysis shows that the hydrodynamic force tending to disrupt the chain has a maximum near the middle point of the chain [1, 8]. The development of the expressions for $F_{\mathrm{r}}^{\mathrm{h}}, F_{\mathrm{r}}^{\mathrm{m}}, \Gamma_{n}^{\mathrm{h}}$, $\Gamma_{n}^{\mathrm{m}}$ is presented in $[8,12]$. In the denotations used in [12], they appear as follows:

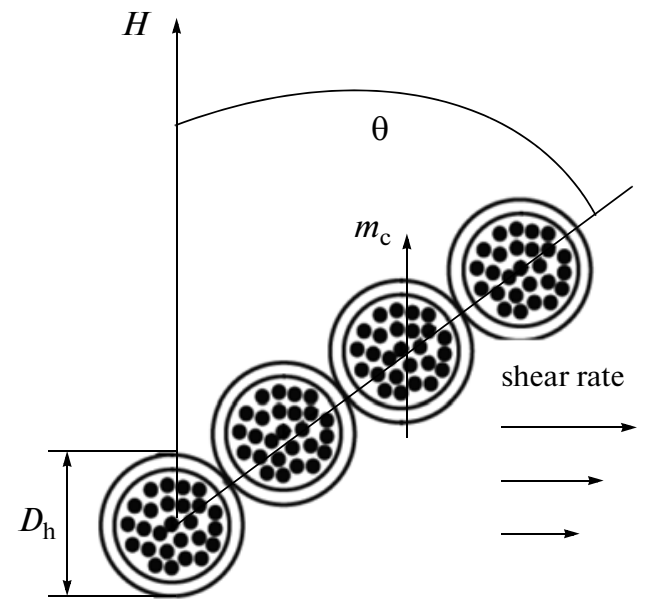

Fig. 3. Schematic representation of a chain consisting of magnetic clusters.

$$
\begin{gathered}
F_{\mathrm{r}}^{\mathrm{h}}=\frac{\dot{\gamma} \beta D_{\mathrm{h}} v(v+1) \sin \theta_{n} \cos \theta_{n}}{2}, \\
F_{\mathrm{r}}^{\mathrm{m}}=\frac{3 \lambda k T\left(3 \cos ^{2} \theta_{n}-1\right)}{D_{\mathrm{h}}}, \\
\Gamma_{n}^{\mathrm{h}}=\frac{\dot{\gamma} \beta D_{\mathrm{h}}^{2} v\left(2 v^{2}+3 v+1\right) \cos ^{2} \theta_{n}}{3}, \\
\Gamma_{n}^{\mathrm{m}}(t)=6(n-1) k T \lambda \sin \theta_{n} \cos \theta_{n}, \\
\beta=3 \pi \eta_{0} D_{\mathrm{h}}, \quad v=\frac{n-1}{2}, \quad \lambda=\frac{\mu_{0} m_{\mathrm{c}}^{2}}{4 \pi D_{\mathrm{h}}^{3} k T} .
\end{gathered}
$$

Here, $\lambda$ is the dimensionless energy of the dipoledipole intercluster interaction. Note that expressions (3) correspond to the approximation in which the field inside a clustered particle is equal to external field $H$ and its magnetic moment is oriented along the external field.

Equating magnetic moment of force $\Gamma_{n}^{\mathrm{m}}$ to hydrodynamic moment of force $\Gamma_{n}^{\mathrm{h}}$ and magnetic attracting force $F_{\mathrm{r}}^{\mathrm{m}}$ to hydrodynamic disrupting force $F_{\mathrm{r}}^{\mathrm{h}}$, we obtain the following set of equations for angle $\theta_{n}$ and number $n_{\mathrm{c}}$ :

$$
\begin{gathered}
\tan \theta_{n}=\frac{\pi s\left(2 v_{\mathrm{c}}^{2}+3 v_{\mathrm{c}}+1\right)}{12 \lambda}, \\
\frac{2-\tan ^{2} \theta_{n}}{\tan \theta_{n}}=\frac{\pi s v_{\mathrm{c}}\left(v_{\mathrm{c}}+1\right)}{2 \lambda}, \quad v_{\mathrm{c}}=\frac{n_{\mathrm{c}}-1}{2},
\end{gathered}
$$

where

$$
s=\frac{\dot{\gamma} \eta_{0} D_{\mathrm{h}}^{3}}{k T}
$$


Through elementary transformations, this set is reduced to the following fourth-order algebraic equation for maximum number of clusters in an intact chain $n_{\mathrm{c}}$ :

$$
v_{\mathrm{c}}^{4}+\frac{21}{8} v_{\mathrm{c}}^{3}+\frac{37}{16} v_{\mathrm{c}}^{2}+\frac{3}{4} v_{\mathrm{c}}+\frac{1}{16}-18 a=0,
$$

where

$$
v_{\mathrm{c}}=\frac{n_{\mathrm{c}}-1}{2}, a=\frac{\lambda^{2}}{\pi^{2} s^{2}} .
$$

Equation (5) has the only real positive root. The other roots of Eq. (5) have no physical meaning. The solution to Eq. (5) is presented in the Appendix.

Under the inertia-free approximation that is valid for real types of the motion of submicrosized particles, a balance occurs between the magnetic moment of force $\Gamma_{n}^{\mathrm{m}}$ and the hydrodynamic moment of force $\Gamma_{n}^{\mathrm{h}}$, which are acting on the chain:

$$
\Gamma_{n}^{\mathrm{m}}=\Gamma_{n}^{\mathrm{h}} .
$$

After the macroscopic flow is stopped (i.e., at $\dot{\gamma}=0$ ), the moment of the hydrodynamic force is determined by the angular rate of rotation of the chain rather than the shear rate of the suspension. Therefore, in formula (3) for hydrodynamic moment of force $\Gamma_{n}^{\mathrm{h}}$, shear rate $\dot{\gamma}$ should be replaced by $-\frac{d \theta_{n}(t)}{d t}$. Taking into account this replacement and equating the magnetic and hydrodynamic moments, we obtain the equation for angle $\theta_{n}$ :

$$
\begin{gathered}
-\frac{\beta D_{\mathrm{h}}^{2} v\left(2 v^{2}+3 v+1\right) \cos \theta_{n}(t)}{3} \frac{d \theta_{n}(t)}{d t} \\
=6(n-1) k T \lambda \sin \theta_{n}(t) .
\end{gathered}
$$

Let, before the flow is stopped, i.e., at $t=0$, the angle between the chain axis and the direction of the magnetic field be equal to $\theta_{n 0}$. The value of this angle can be determined from the first of Eqs. (4):

$$
\tan \theta_{n 0}=\frac{\pi s\left(2 v^{2}+3 v+1\right)}{12 \lambda} .
$$

The solution of Eq. (6) with initial condition (7) has the following form:

$$
\begin{gathered}
\sin \theta_{n}(t)=\sin \theta_{n 0} \exp \left(-\frac{t}{\tau_{n}}\right), \\
\tau_{n}=\frac{\beta D_{\mathrm{h}}^{2}\left(2 v^{2}+3 v+1\right)}{36 k T \lambda} .
\end{gathered}
$$

Here, $\tau_{n}$ has the meaning of the relaxation time.
Taking into account relation (2), the expressions for symmetric $\sigma^{\mathrm{s}}$ and antisymmetric $\sigma^{\mathrm{a}}$ components of stress $\sigma$ can be written as follows:

$$
\begin{gathered}
\sigma^{\mathrm{s}}(t)=\eta_{0} V_{\mathrm{h}} \\
\times \sum_{n=2}^{n_{c}} \frac{\beta_{n}\left[1-2 \sin ^{2} \theta_{n 0} \exp \left(-\frac{2 t}{\tau_{n}}\right)\right] \sin \theta_{n 0} \exp \left(-\frac{t}{\tau_{n}}\right) n g_{n}}{\tau_{n} \sqrt{1-\sin ^{2} \theta_{n 0} \exp \left(-\frac{2 t}{\tau_{n}}\right)}}, \\
\sigma^{\mathrm{a}}(t)=3 k T \lambda \sum_{n=2}^{n_{\mathrm{c}}}(n-1) \sin \theta_{n 0} \\
\times \exp \left(-\frac{t}{\tau_{n}}\right) \sqrt{1-\sin ^{2} \theta_{n 0} \exp \left(-\frac{2 t}{\tau_{n}}\right)} g_{n} .
\end{gathered}
$$

The function of distribution over the number of magnetizable particles in a chain $g_{n}$ was assessed in [12] and successfully used in [3] to describe stationary magnetoviscous effects in magnetic fluids containing clustered particles.

Using the results of [12], we obtain the following:

$$
g_{n}=\frac{X^{n} \exp \left[-\lambda\left(3 \cos ^{2} \theta_{n 0}-1\right)\right]}{V_{\mathrm{h}}},
$$

where $X^{n}$ is the Lagrange factor, which is determined from the normalization condition,

$$
\sum_{n=1}^{n_{c}} n g_{n}=\frac{\varphi_{\mathrm{h}}}{V_{\mathrm{h}}} .
$$

Here, $\varphi_{\mathrm{h}}$ is the hydrodynamic concentration of clusters in a ferrofluid.

The experimental and theoretical time dependences of the stress after the instantaneous termination of the flow of the clustered magnetic fluid are shown in Figs. 2a and 2b. At rather short and intermediate $(\sim 100 \mathrm{~s})$ times $t$ elapsed after the termination of the flow, the experimental and theoretical results are in rather good agreement. This finding testifies that the finite rate of variations in angle $\theta$ of the orientation of the chain with respect to the field is adequate to the observed relaxation phenomena. Note that other physically possible microscopic mechanisms responsible for the stress relaxation in magnetic fluids have been discussed in review [9].

The experimental and theoretical behaviors of stress $\sigma(t)$ differ significantly from each other at longer times $t$. According to the experimental data, stress tends asymptotically to finite value $\sigma_{\mathrm{r}}$ at $t \rightarrow \infty$; however, the chain model predicts that $\sigma$ will tend to zero.

As has been mentioned above, finite residual stress $\sigma_{\mathrm{r}}$ can be related to dense large aggregates, which are formed along with the chains. When the flow is 
stopped, these aggregates can block the measuring cell and generate stationary stress $\sigma_{\mathrm{r}}$.

\section{BULKY AGGREGATES}

Dense large aggregates consisting of magnetizable composite particles become oblong in a magnetic field. As a first approximation, these aggregates can be considered ellipsoids of revolution.

A theory of static mechanical stress generated by a system of ellipsoidal aggregates that block the channel of a measuring unit was developed in [15] (see also [9]). According to this theory, stress $\sigma$ arising in a magnetic suspension at stationary shear $\gamma$. can be written as follows:

$$
\sigma=\frac{1}{V} \frac{\partial F}{\partial \gamma}, \gamma=\tan \theta
$$

Here, $F$ and $V$ are the free energy and volume of a magnetic fluid and $\theta$ is the angle between the axis of an ellipsoid of revolution and the direction of a magnetic field.

In uniform external magnetic field $H$, the free energy of the magnetic fluid containing large aggregates can be represented as $[15,16]$

$$
F=-\mu_{0} \int_{0}^{H} m_{z} d H
$$

where

$$
m_{z}=N_{\mathrm{a}} m_{z \mathrm{a}} .
$$

Here, $m_{z}$ is the projection of the total magnetic moment of the suspension onto the axis of the magnetic field ( $z$ axis), $N_{a}$ is the number of large droplets in the system, and $m_{z \mathrm{a}}$ is the projection of the magnetic moment of a large aggregate onto the axis of the magnetic field. The value of $m_{z \mathrm{a}}$ can obviously be resolved into components $m_{\| \mathrm{a}}$ and $m_{\perp \mathrm{a}}$ that are parallel and perpendicular to the ellipsoid axis:

$$
m_{z \mathrm{a}}=m_{\| \mathrm{a}} \cos \theta+m_{\perp \mathrm{a}} \sin \theta, \quad m_{z \mathrm{a}}=\frac{m_{\mathrm{IIa}}+m_{\perp \mathrm{a}} \gamma}{\sqrt{1+\gamma^{2}}} .
$$

The components of the magnetic moment of the ellipsoid of revolution can be expressed via magnetization components $M_{\| \mathrm{a}}, M_{\perp \mathrm{a}}$ and volume of the ellipsoid of revolution $V_{\mathrm{a}}$ as follows:

$$
m_{\| \mathrm{a}}=M_{\| \mathrm{a}} V_{\mathrm{a}}, \quad m_{\perp \mathrm{a}}=M_{\perp \mathrm{a}} V_{\mathrm{a}} .
$$

The following important relations result from [16]:

$$
H_{\|}^{(i)}+M_{\|} n_{\|}=H_{\|}, \quad H_{\perp}^{(i)}+M_{\perp} n_{\perp}=H_{\perp} .
$$

Here, $H_{\|}^{(i)}$ and $H_{\perp}^{(i)}$ are the components of the magnetic field strength inside a large droplet; $n_{\|}$and $n_{\perp}$ are the demagnetization factors of the ellipsoid of revolution; and $H_{\|}$and $H_{\perp}$ are the components of the external magnetic field strength, which can easily be represented via the external magnetic field strength as follows:

$$
\begin{aligned}
& H_{\|}=H \cos \theta, \quad H_{\perp}=H \sin \theta, \\
& H_{\|}=\frac{H}{\sqrt{1+\gamma^{2}}}, \quad H_{\perp}=\frac{H \gamma}{\sqrt{1+\gamma^{2}}} .
\end{aligned}
$$

In accordance with the data on bulky structures in suspensions containing magnetizable particles (see, e.g., [9]), we assume that the ellipsoids of revolution are strongly stretched; hence, the expressions for the demagnetization factors acquire the following simple form:

$$
n_{\|} \approx 0, \quad n_{\perp} \approx \frac{1}{2}
$$

The magnetization components of an ellipsoidal aggregate can be written as

$$
M_{\|}=\chi H_{\|}^{(i)}, \quad M_{\perp}=\chi H_{\perp}^{(i)} .
$$

Here, $\chi$ is the magnetic susceptibility of an aggregate. Generally speaking, this value depends on field $H^{(i)}$ inside the aggregate, which is, in turn, determined by external magnetic field strength $H$ and shear value $\gamma$. The following expressions are obtained by combining Eqs. (12)-(14):

$$
\begin{gathered}
H_{\perp}=\frac{2 \gamma H}{(\chi+2) \sqrt{1+\gamma^{2}}}, \\
M_{\|}=\frac{\chi H}{\sqrt{1+\gamma^{2}}}, \quad M_{\perp}=\frac{2 \gamma H \chi}{(\chi+2) \sqrt{1+\gamma^{2}}} .
\end{gathered}
$$

As a first approximation, magnetization modulus $|M|$ of an aggregate can be estimated based on the Langevin formula:

$$
|M|=\frac{N_{\mathrm{p}} \Phi_{\mathrm{c}}}{V_{\mathrm{h}}}\left[m_{\mathrm{p}} \operatorname{cth}\left(\frac{\mu_{0} m_{\mathrm{p}}\left|H^{(i)}\right|}{k T}\right)-\frac{k T}{\mu_{0}\left|H^{(i)}\right|}\right] .
$$

Here, $\Phi_{c}$ is the hydrodynamic concentration of clustered particles inside the ellipsoid of revolution.

It is obvious that

$$
|M|=\sqrt{M_{\|}^{2}+M_{\perp}^{2}},\left|H^{(i)}\right|=\sqrt{\left(H_{\|}^{(i)}\right)^{2}+\left(H_{\perp}^{(i)}\right)^{2}} .
$$

By combining Eqs. (15)-(17), we derive a transcendental equation for $\chi$ : 

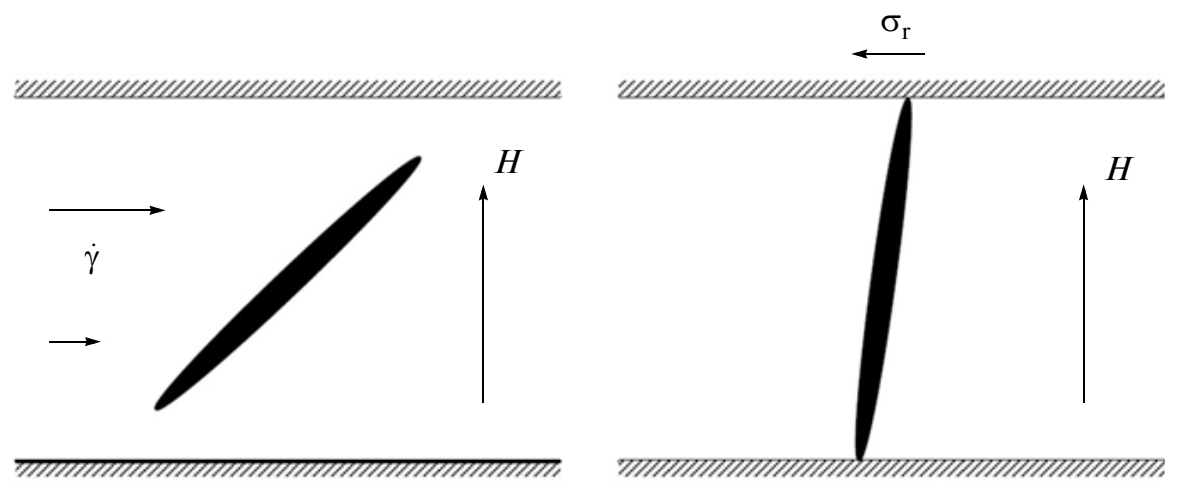

Fig. 4. Mechanism of residual stress development.

$$
\begin{gathered}
=\frac{\frac{\chi H V_{\mathrm{h}}}{(\chi+2) N_{\mathrm{p}} \Phi_{\mathrm{c}}}}{m_{\mathrm{p}} \sqrt{1+\gamma^{2}} \operatorname{cth}\left(\frac{\mu_{0} m_{\mathrm{p}} H \sqrt{\chi^{2}+4 \chi+4 \gamma^{2}+4}}{k T(\chi+2) \sqrt{1+\gamma^{2}}}\right)} \\
-\frac{k T(\chi+2)\left(1+\gamma^{2}\right)}{{\sqrt{\chi^{2}+4 \chi+4 \gamma^{2}+4}}_{\mu_{0} H\left(\chi^{2}+4 \chi+4 \gamma^{2}+4\right)}}
\end{gathered}
$$

which can be numerically solved.

By combining Eqs. (9)-(11) and (15), we obtain the following expressions for energy $F$ and static residual stress $\sigma$ :

$$
\begin{gathered}
F=-\frac{\mu_{0} N_{\mathrm{a}} V_{\mathrm{a}}}{1+\gamma^{2}} \int_{0}^{H} \frac{H \chi\left(\chi+2 \gamma^{2}+2\right)}{\chi+2} d H, \\
\sigma=-\mu_{0} \Psi \frac{\partial}{\partial \gamma}\left[\frac{1}{1+\gamma^{2}} \int_{0}^{H} \frac{H \chi\left(\chi+2 \gamma^{2}+2\right)}{\chi+2} d H\right],
\end{gathered}
$$

where

$$
\Psi=\frac{N_{\mathrm{a}} V_{\mathrm{a}}}{V}, \quad \Psi=\frac{p \varphi}{\Phi_{\mathrm{c}}} .
$$

Here, $\Psi$ is the hydrodynamic volume concentration of large droplets and $p$ is the fraction of the magnetic material in the large clusters.

Stress $\sigma$ as a function of $\gamma$ has maximum $\sigma_{\max }$ at some shear value $\gamma_{\mathrm{cr}}$. According to the theory [15], $\sigma_{\max }$ corresponds to the static yield stress $\sigma_{\mathrm{s}}$ of the magnetic fluid. When the external shear stress exceeds $\sigma_{\mathrm{s}}$, the system of aggregates cannot statically compensate for this stress, so the magnetic suspension starts to flow, which is accompanied by detachment of aggregates from the channel walls. Under the impact of the flow, the aggregates strongly deviate from the direction of the magnetic field, which is perpendicular to the channel walls; hence, the projections of their axes onto this direction become smaller than the channel width.

After the flow is stopped, the aggregates are oriented in parallel to the field and block the experimental unit. As a result, residual stress $\sigma_{\mathrm{r}}$. arises. The mechanism of the emergence of the residual stress is illustrated in Fig. 4.

If the disruption of the large aggregates in the moving magnetic fluid is ignored, the residual stress must be equal to $\sigma_{s}$. If the aggregates are disrupted under the flow conditions and become shorter than those at the onset of the movement, the residual stress must be somewhat lower than the static yield stress.

Experimental data on the static yield stress and residual stress are shown in Fig. 5. It can be seen that the static yield stress does somewhat exceed the residual stress; however, they are of the same order of magnitude. Unfortunately, a commonly accepted theory of the disruption of bulky clusters in magnetic fluids under the conditions of a shear flow has not been developed. In order to obtain physically clear results, we ignore the disruption of these aggregates in the moving medium. The foundation for this is the closeness of the measured static yield stress and residual stress.

Experimental and theoretical residual stress values are compared in Fig. 6. The only fitting parameter $p$ has been used in the calculations. The theoretical results shown in Fig. 6 correspond to value $p=0.12$, which indicates that the majority of the clustered particles are assembled into chains and only a small portion of the particles form large aggregates. This result confirms the previous assumption that the rheological properties of a magnetic fluid in the state of a developed flow are mainly determined by chain-shaped aggregates, while the bulky structures are responsible 


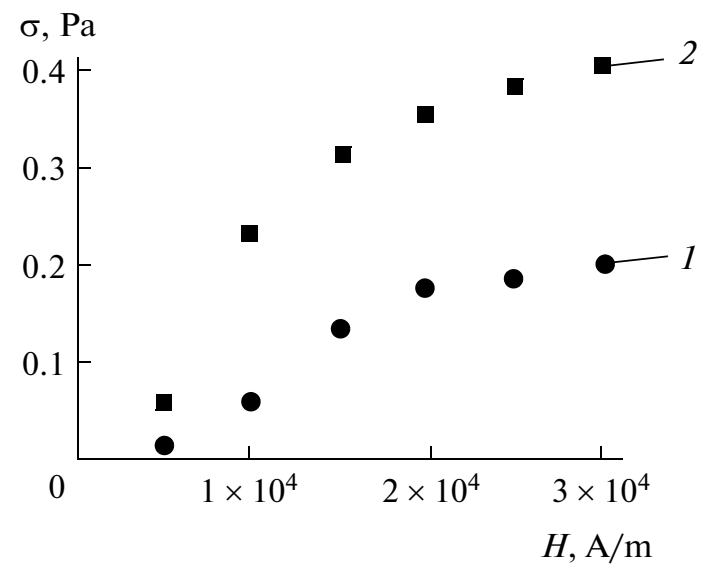

Fig. 5. (1) Residual stress and (2) yield stress of clustered ferrofluid as functions of magnetic field strength.

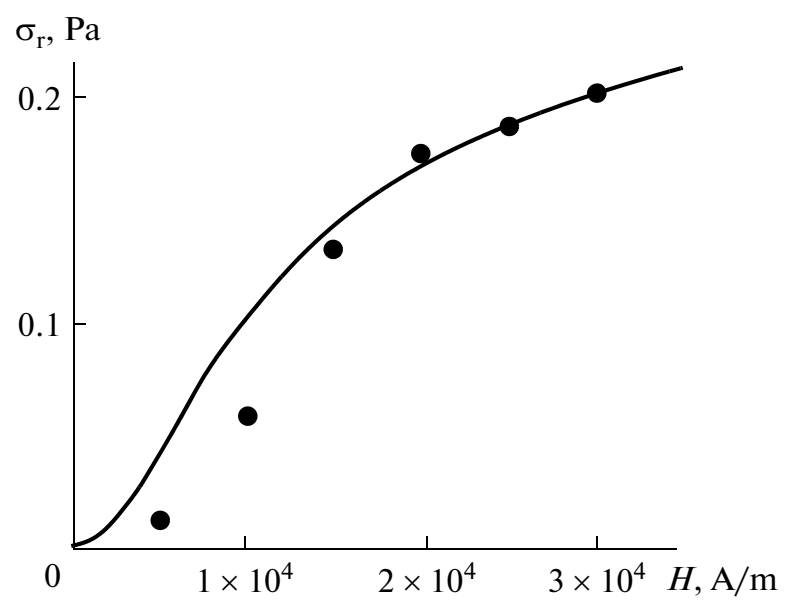

Fig. 6. (1) Theoretical and (2) experimental dependences of residual stress on magnetic field strength. The fraction of magnetic material is $p=0.12$.

single-domain ferroparticles in them, a more exact coincidence between the theoretical and experimental data can hardly be expected.

\section{CONCLUSIONS}

Magnetic fluids containing clustered particles combine the sedimentation stability with high sensitivity of rheological parameters to magnetic fields. Experiments have demonstrated that the rheological properties of the cluster fluids are distinguished by a number of significant features, namely, a long-term stress relaxation and a residual stress emerging after the flow is stopped.

The observed effects can be explained under the assumption that the clustered particles are capable of forming both chains and dense large aggregates. In a well-developed flow, both the chains and ellipsoidal droplets are tilted at a certain angle to the external field. The tilt angle is determined by the combination of the moments of the hydrodynamic and magnetic forces acting on the aggregates. Chain-shaped aggregates have the predominant effect on the rheology of the examined fluid under the flow conditions; after the flow rate is changed, the finite rate of variations in the orientation angle of the chains causes the effect of the long-term stress relaxation that have been observed.

After cessation of the flow, the large droplets tend be oriented along the field, thus blocking the channel and generating a constant residual stress. The proposed model, which is based on the aforementioned assumptions, quantitatively describes the experimental results on the order of magnitude. 

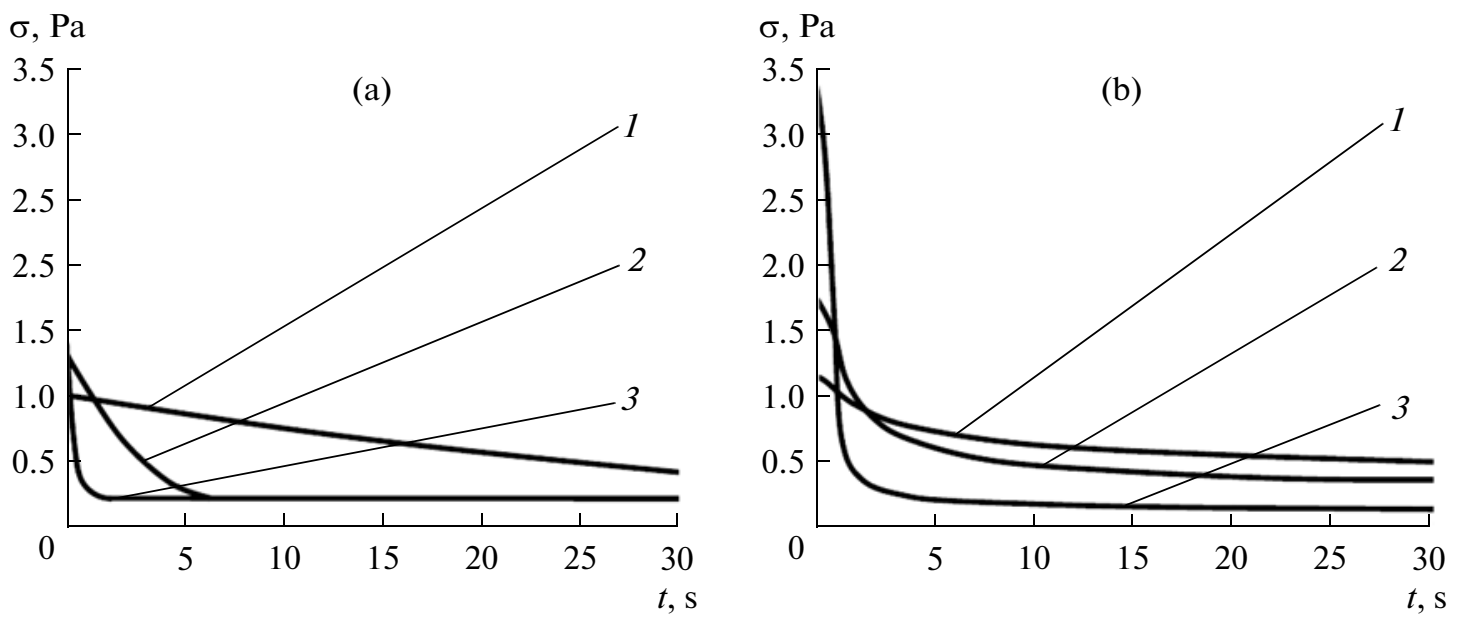

Fig. 7. (a) Experimental and (b, c) theoretical time dependences of stress at magnetic field stress $H=30 \mathrm{kA} / \mathrm{m}$ and different shear rates: (1) $0 \leq \dot{\gamma} \leq 0.02 \mathrm{~s}^{-1}$; (2) $0 \leq \dot{\gamma} \leq 0.2 \mathrm{~s}^{-1}$; (3) $0 \leq \dot{\gamma} \leq 2 \mathrm{~s}^{-1}$.

\section{APPENDIX: PARAMETERS OF RELAXATION EQUATIONS}

Kinetic coefficient $\beta_{n}$ was determined in [13] as follows:

$$
\beta_{n}=\frac{2\left(n^{2}-1\right)}{n\left(n^{2} \alpha_{0}+\beta_{0}\right)},
$$

where

$$
\begin{gathered}
\alpha_{0}=\frac{2}{3} \text { at } n=1, \\
\alpha_{0}=-\frac{1}{n^{2}-1}\left[\frac{2}{n}+\frac{1}{\sqrt{n^{2}-1}} \ln \left(2 n^{2}-1-2 n \sqrt{n^{2}-1}\right)\right] \\
\text { at } n>1, \\
\beta_{0}=\frac{2}{3} \text { at } n=1, \\
\beta_{0}=\frac{1}{n^{2}-1}\left[n-\frac{1}{2 \sqrt{n^{2}-1}} \ln \left(2 n^{2}-1+2 n \sqrt{n^{2}-1}\right)\right] \\
\text { at } n>1 .
\end{gathered}
$$

The solution to fourth-order equation (5) is reduced to solving the auxiliary cubic equation using the Ferrari method:

$$
\begin{gathered}
v_{\mathrm{c}}=\sqrt{\frac{A_{0}}{2}}+\sqrt{\frac{1}{2}\left(\frac{99}{4096 \sqrt{2 A_{0}}}+\frac{139}{512}-A_{0}\right)}-\frac{21}{32}, \\
A_{0}=\frac{9801(720576 a-343)}{512 B_{0}},
\end{gathered}
$$

$$
\begin{gathered}
B_{0}=3(524288 a+251)(720576 a-343) \\
+8192(15372288 a-4165)\left(\beta_{1}^{2}+\beta_{2}^{2}\right) \\
+10494148608\left(\beta_{1}^{4}+\beta_{2}^{4}\right) \\
\beta_{1}=\sqrt[3]{-\frac{q}{2}+\sqrt{\frac{q^{2}}{4}+\frac{p^{3}}{27}}}, \quad \beta_{2}=\sqrt[3]{-\frac{q}{2}-\sqrt{\frac{q^{2}}{4}+\frac{p^{3}}{27}}} \\
q=\frac{1}{256}\left(417 a-\frac{343}{1728}\right), \quad p=18 a-\frac{49}{3072} .
\end{gathered}
$$

\section{ACKNOWLEDGMENTS}

This work was supported by the Russian Foundation for Basic Research (projects nos. 10-0196002r_ural_a, 10-02-00034-a, 10-02-96001r_ural_a, and 12-01-00132-a) and Ural Federal University in the framework of the Program for Support of Young Researchers.

\section{REFERENCES}

1. Odenbach, S., Magnetoviscous Effects in Ferrofluids. Lect. Notes Phys., Berlin: Springer, 2002.

2. Dutz, S., Andrä, W., Hergt, R., Müler, R., Oestreich C., Schmidt, C., Töpfer, J., Zeisberger, M., and Bellemann, M., J. Magn. Magn. Mater., 2007, vol. 311, p. 51 .

3. Borin, D., Zubarev, A., Chirikov, D., Muller, R., and Odenbach, S., J. Magn. Magn. Mater., 2011, vol. 323, p. 1273.

4. Zubarev, A.Yu., Chirikov, D.N., Borin, D.Yu., and Odenbach, S., Abstracts of Papers, 15 Mezhdunar. Plesskaya nauchn. konf. po nanodispersnym magnitnym zhidkostyam (15 Int. Ples Sci. Conf. on Nanodisperse Magnetic Fluids), Ivanovo: Ivanovsk. Gos. Energ. Univ., 2012, p. 113. 
5. Shliomis, M.I., Usp. Fiz. Nauk, 1974, vol. 112, p. 427.

6. Ilg, P. and Odenbach, S., in Colloidal Magnetic Fluids, Odenbach, S., Ed., Berlin: Springer, 2009, p. 249.

7. Zubarev, A.Yu., Fleisher, J., and Odenbach, S., Physica $A$ (Amsterdam), 2005, vol. 358, p. 475.

8. Martin, J. and Andreson, R., J. Chem. Phys., 1996, vol. 104, p. 4814.

9. Bossis, G., Volkova, O., Lacis, S., and Meunier, A., in Ferrofluids: Magnetically Controllable Fluids and Their Applications, Odenbach, S., Ed., Berlin: Springer, 2002, p. 202.

10. Islam, M.F., Lin, K.H., Lacoste, D., Lubenski, T.C., and Yodh, A.G., Phys. Rev. E: Stat. Phys., Plasmas, Fluids, Relat. Interdiscip. Top., 2003, vol. 67, p. 021402.
11. Iskakova, L.Yu., Romanchuk, A.P., and Zubarev, A.Yu., Physica A (Amsterdam), 2006, vol. 366, p. 18.

12. Zubarev, A.Yu. and Iskakova, L.Yu., Physica $A$ (Amsterdam), 2007, vol. 382, p. 378.

13. Pokrovskii, V.N., Statisticheskaya gidromekhanika razbavlennykh suspenzii (Statistical Hydromechanics of Dilute Suspensions), Moscow: Nauka, 1978.

14. Zubarev, A.Yu. and Iskakova, L.Yu., Phys. Rev. E: Stat. Phys., Plasmas, Fluids, Relat. Interdiscip. Top., 2000, vol. 61, p. 5415.

15. Bossis, G., Lemaire, E., Volkova, O., and Clerix, H., J. Rheol. (N. Y.), 1997, vol. 41, p. 687.

16. Landau, L., Lifshitz, E., and Pitaevski, L., Electrodynamics of Continuous Media, Oxford: Pergamon, 1984.

Translated by D. Terpilovskaya 